\title{
Myositis Associated with Infratemporal Space Abscess in Patient with Myxofibrosarcoma of Nasal Cavity: Case Report
}

\author{
Jiyeon Kim, Min Chang, YounJung Park, Hyung-Joon Ahn, \\ Seong-Taek Kim, Jong-Hoon Choi, Jeong-Seung Kwon \\ Department of Orofacial Pain and Oral Medicine, Dental Hospital, Yonsei University College of Dentistry, Seoul, Korea
}

Received September 6, 2021

Revised September 19, 2021

Accepted September 19, 2021

\author{
Correspondence to: \\ Jeong-Seung Kwon \\ Department of Orofacial Pain and Oral \\ Medicine, Dental Hospital, Yonsei University \\ College of Dentistry, 50-1 Yonsei-ro, \\ Seodaemun-gu, Seoul 03722, Korea \\ Tel: +82-2-2228-3111 \\ Fax: +82-2-393-5673 \\ E-mail: jskwon@yuhs.ac \\ https://orcid.org/0000-0003-4584-7355
}

The limited mouth opening, also known as trismus, can result from temporomandibular joint disorders, infection, neoplasm, trauma, and abnormal anatomic structure like coronoid hyperplasia. Head and neck cancer patients often complain of limited mouth opening, which is usually induced by myofibrotic contracture of masticatory muscle. But clinicians should consider any reasons such as infection or cancer growth and metastasis if trismus gets worse or pain develops. We report the case of the patient, who was diagnosed with myxofibrosarcoma on nasal cavity. He had suffered from trismus after concurrent chemoradiotherapy. However, pain had developed and trismus had worsened. He was diagnosed with infratemporal space abscess and myositis of masticatory muscles.

Key Words: Head and neck neoplasms; Myositis; Trismus

\section{INTRODUCTION}

The limited mouth opening (LMO) is a condition in which the mouth opening is limited owing to various causes, including trauma, infection, neurologic disorders, neoplasms, anatomic structures, and temporomandibular joint disorders [1-3]. Head and neck cancer patients often suffer from trismus, especially after radiation therapy. It is known that the main reason to cause trismus is myofibrotic masticatory muscle contracture, which occurs as a result of radiationinduced fibrosis of muscles [4-8].

In these patients, clinicians should consider not only muscle contracture but also myositis due to infection as a cause of LMO because they are immunocompromised. Myositis is an inflammatory muscle disease, which has clinical characteristics of inflammation or infection such as edema, erythema, and increased temperature.

In this paper, we present the case of a patient, who already had the LMO after concurrent chemoradiotherapy
(CCRT) for myxofibrosarcoma (MFS) of nasal cavity. However, pain had developed and the LMO had worsened. He was diagnosed with infratemporal space abscess and myositis of masticatory muscles.

The protocol of this study was approved by the Institutional Review Board of Yonsei University Dental Hospital (IRB no. 2-2021-0090).

\section{CASE REPORT}

A 41-year-old man visited the Department of Orofacial Pain and Oral Medicine of Yonsei University Dental Hospital (Seoul, Korea) with a complaint of pain in his right masseter area and LMO.

He underwent partial removal of extensive MFS arising in right nasal cavity by the otolaryngologist 4 years and 6 months before the visit. He had also received CCRT for 5 weeks after surgery and kept taking an anti-cancer medication Pazopanib (Votrient; Novartis, Ljubljana, Slovenia). 
Although he had experienced some discomfort and limitation during opening mouth after CCRT, he started to feel pain in right masseter area when opening mouth and chewing and LMO worsened 2 months before the visit without any reason. Even though he had already taken strong analgesics (Targin CR [oxycodone hydrochloride and naloxone hydrochloride], IRcodon [oxycodone hydrochloride], Mypol [acetaminophen/codeine phosphate hydrate/ibuprofen]), his pain had aggravated. He did not show any inflammatory signs in orofacial area and blood tests including complete blood count, routine chemistry were normal except low red blood cell count and hemoglobin.

On clinical examination, the unassisted maximal interincisal distance was about $24 \mathrm{~mm}$ with pain in right masseter area. The assisted maximal interincisal distance was $26 \mathrm{~mm}$ with hard end feel. The maximum range of protrusive, right lateral, and left lateral movement were $4 \mathrm{~mm}, 2 \mathrm{~mm}$, and 3 $\mathrm{mm}$ respectively. The protrusive and right lateral movement induced pain in both and right temporomandibular joint pain respectively. The palpation revealed familiar pain in the origin of the right masseter. The occlusal contacts were stable in maximum intercuspation. The panoramic radiograph on the first visit revealed that right maxillary sinus walls were extensively disappeared and haziness in right maxillary sinus was noted (Fig. 1).

Based on history and clinical examinations, the patient was diagnosed with a myofascial pain on right masseter and myofibrotic contracture due to radiation therapy. $\mathrm{He}$ underwent physical therapy, and practiced mouth opening exercise with wooden tongue depressors. However, symptoms did not improve. Cone beam computed tomography revealed that right maxillary sinus was occupied with radiopaque lesion infiltrated to surrounding structures without pathologic findings in mandible condyle (Fig. 2). We looked at again the post-operative magnetic resonance (MR) images taken at the department of oncology for follow-up purpose and they revealed high signal intensity with diffuse inflammatory change on infratemporal space and right temporalis, and lateral pterygoid muscle (Fig. 3). Therefore, he was diagnosed with myositis caused by infratemporal space abscess, and then he was referred to oncologist for evaluation and further treatment.

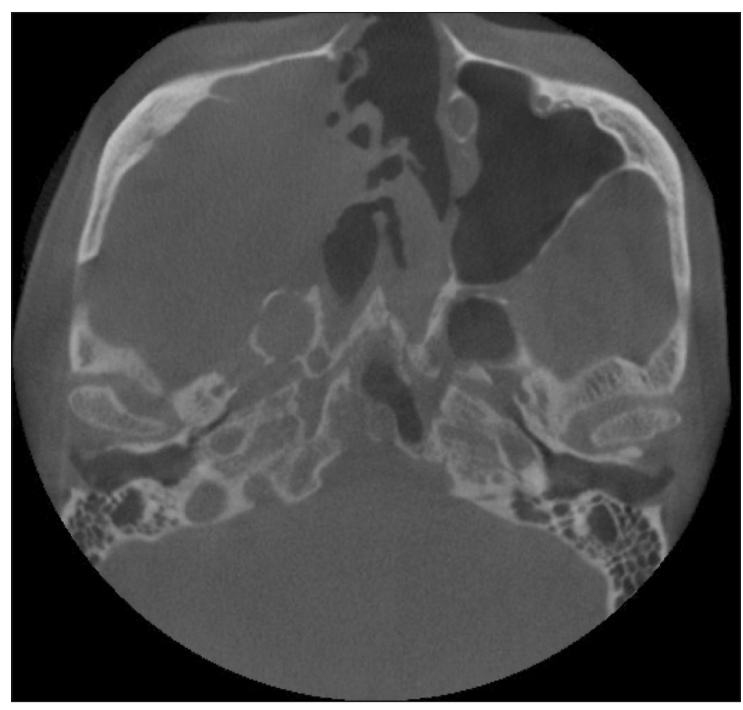

Fig. 2. Axial view of cone beam computed tomography on the first visit. Destruction of right maxillary sinus posterior wall was shown, but no definite pathologic bony change on both condyle was found.
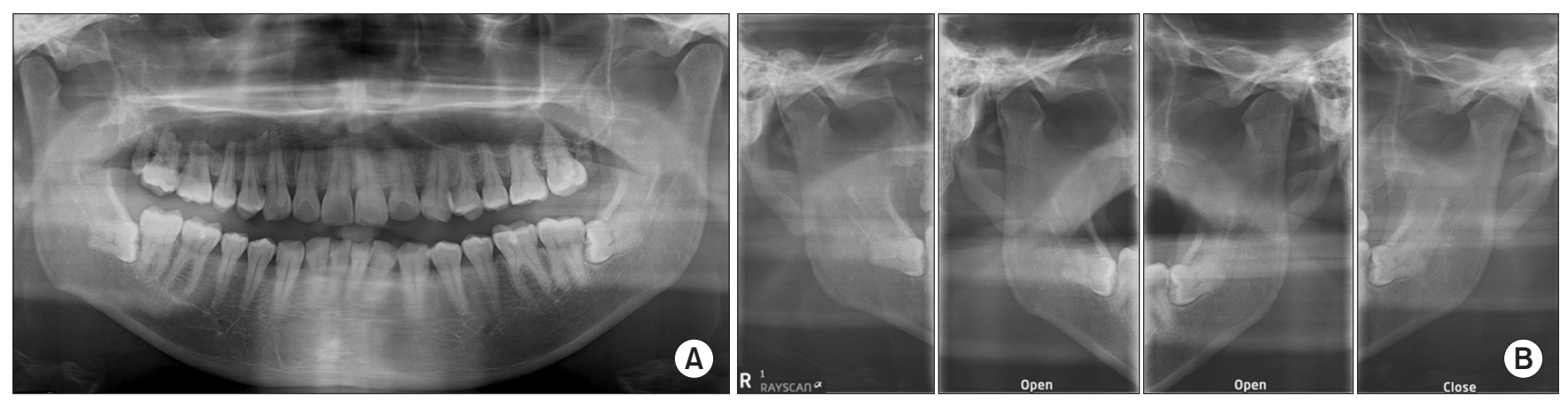

Fig. 1. Panoramic radiograph on the first visit. (A) Destruction of right maxillary sinus wall and haziness in right sinus were noted. (B) Panoramic temporomandibular joint radiograph revealed limitation of condylar translation in both temporomandibular joints. 



Fig. 3. Axial T2-weighted magnetic resonance images taken at the department of oncology. (A) Two months before the first visit. It revealed high signal intensity on right infratemporal space, temporalis (arrowhead), and lateral pterygoid muscle (arrows). (B) 3 days after first visit. It showed more increased high signal intensity with diffuse inflammatory change compared to 2 months before. A, anterior view; $P$, posterior view; $R$, right view; $L$, left view.

\section{DISCUSSION}

Myositis, or muscle inflammation is characterized by the symptoms such as swelling, redness and local heat on musculoskeletal area [9]. Most common etiologies are infectious agents including picornaviruses (coxsackievirus), echovirus, retroviruses (human immunodeficiency virus, human T-cell lymphotropic virus type 1), borrelia, toxoplasma, and adenoviruses. Nevertheless, non-infectious etiologies such as foods, drugs (D-penicillamine, cimetidine), occupational exposure, and medical devices should also be considered [9]. In addition, oral surgery or an intramuscular injection sometimes lead to infection of masticatory muscles as a post-operative complication [10].

Myositis of the masticatory muscles causes the limitation of the mandibular movement mainly due to pain. In head and neck cancer patient, it can be assumed that cancer and cancer treatments such as radiotherapy and chemotherapy induce immunosuppressed condition, which causes an infectious myositis. The myositis can exacerbate trismus and pain.

In this case, we took a closer look at MR images before the first visit because pain had developed and the LMO had worsened. Upon T2 images 2 years and 8 months before the first visit, fatty layer of right lateral pterygoid muscle showed significant atrophic change compared to the opposite side (Fig. 4). The fatty layer showed further atrophic change in the MR image taken 1 year and 10 months before the visit (Fig. 5), suggesting the possibility of the post-operative inflammation [11,12]. Whole-body positron

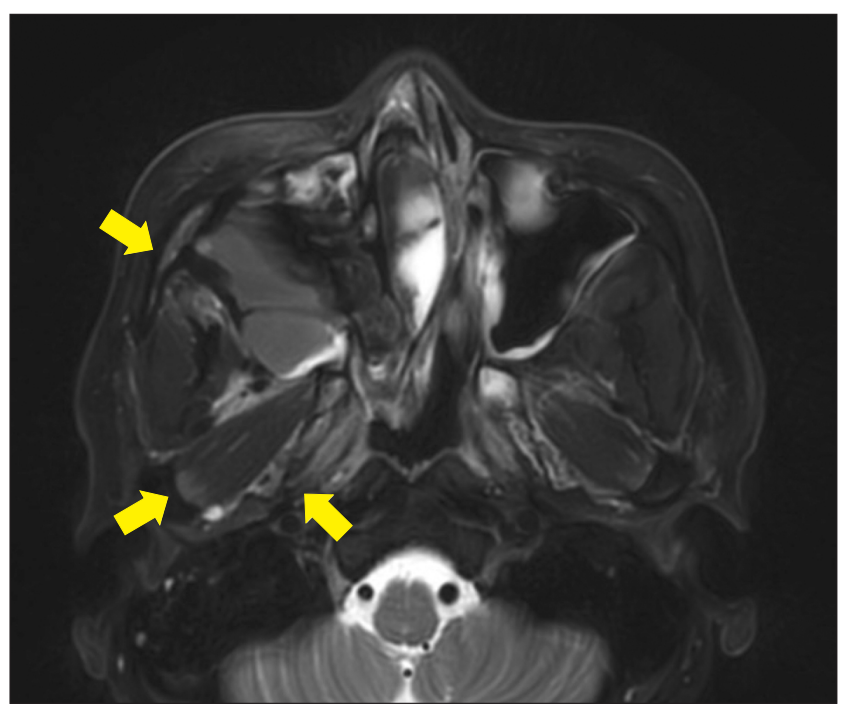

Fig. 4. Axial T2-weighted magnetic resonance images 2 years and 8 months before the first visit. It revealed loss of the fat plane signal (arrows) adjacent to the right lateral pterygoid muscle, indicating more inflammatory changes compared to the left side.

emission tomography/computed tomography did not reveal other lesions. Furthermore, it showed more hyperintense T2-weighted signals compared to the previous imaging. Therefore, it was presumed that recent occurrence of the pain and aggravated LMO were caused by myositis associated with infratemporal space abscess related to known malignancy.

Since there are a number of various causes of the LMO (Fig. 6), the clinicians should consider all possible causes in patient with the LMO. In addition, the clinicians should also be aware that the LMO can be caused by more than one factor. In patient who have head and neck cancer and 


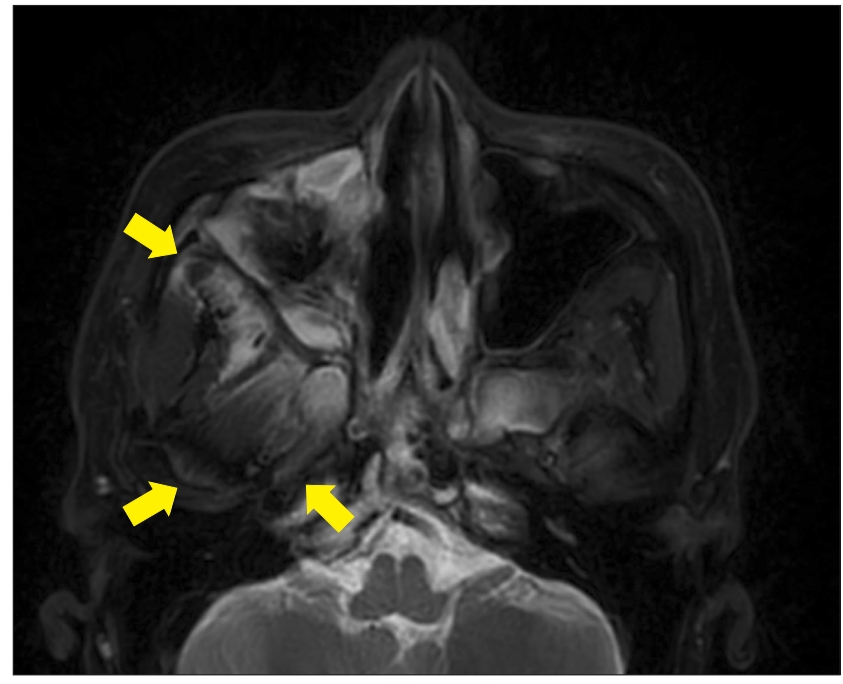

Fig. 5. Axial T2-weighted magnetic resonance images 1 years and 10 months before the first visit. It revealed more loss of the fat plane signal (arrows), compared to 2 years and 8 months before the first visit. It suggested more aggravated inflammation as postoperative side effects. undergo radiotherapy, the LMO is most commonly caused by myofibrotic contracture $[4-8,13]$, but the possibility of infectious myositis also has to be ruled out considering immunocompromised state of the patients. The infectious myositis should also be suspected, especially if the LMO becomes worse, or the pain occurs or changes into a different pattern than before. In such cases, MR or contrast computed tomography images should be taken for accurate diagnosis.

\section{CONFLICT OF INTEREST}

No potential conflict of interest relevant to this article was reported.

\section{ORCID}

Jiyeon Kim

https://orcid.org/0000-0002-2150-6882

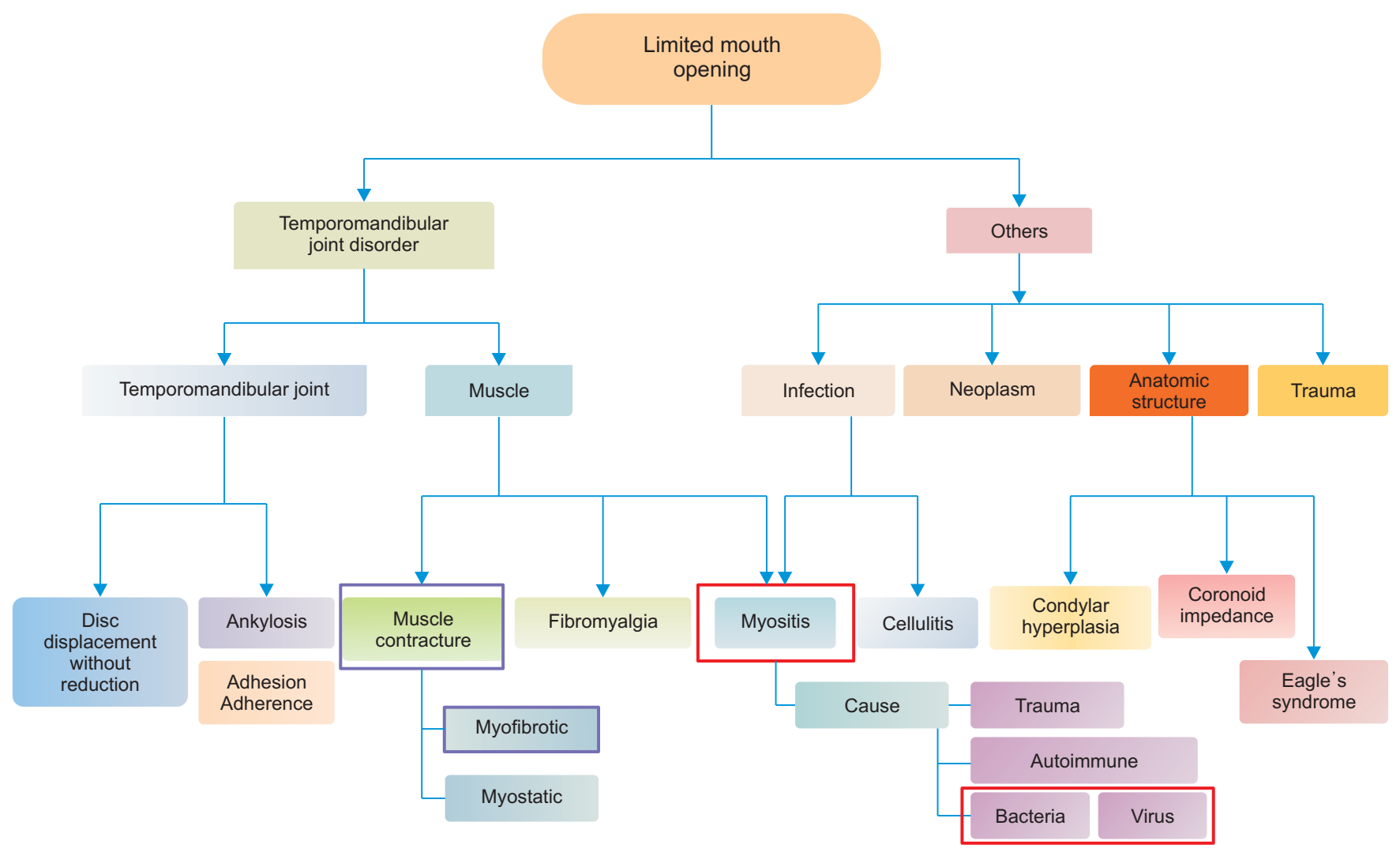

Cancer patient

Fig. 6. Differential diagnosis of limited mouth opening. In this case, limited mouth opening was suspected to be caused by myofibrotic contracture due to radiotherapy and myositis associated with infratemporal space abscess related to known malignancy. 


\author{
Min Chang \\ https://orcid.org/0000-0002-3337-9593 \\ YounJung Park \\ https://orcid.org/0000-0002-9152-7849 \\ Hyung-Joon Ahn \\ https://orcid.org/0000-0001-9669-9781 \\ Seong-Taek Kim \\ https://orcid.org/0000-0001-9506-5103 \\ Jong-Hoon Choi \\ https://orcid.org/0000-0003-3211-3619 \\ Jeong-Seung Kwon \\ https://orcid.org/0000-0003-4584-7355
}

\section{REFERENCES}

1. Satheeshkumar PS, Mohan MP, Jacob J. Restricted mouth opening and trismus in oral oncology. Oral Surg Oral Med Oral Pathol Oral Radiol 2014;117:709-715.

2. Dhanrajani PJ, Jonaidel 0. Trismus: aetiology, differential diagnosis and treatment. Dent Update 2002;29:88-92, 94.

3. Okeson JP. Diagnosis of temporomandibular disorders. In: Okeson JP, ed. Management of temporomandibular disorders and occlusion. 8th ed. St. Louis: Elsevier Health Sciences; 2019. pp. 223258.

4. Bengtson BP, Schusterman MA, Baldwin BJ, et al. Influence of prior radiotherapy on the development of postoperative complications and success of free tissue transfers in head and neck cancer reconstruction. Am J Surg 1993;166:326-330.
5. Dijkstra PU, Huisman PM, Roodenburg JL. Criteria for trismus in head and neck oncology. Int J Oral Maxillofac Surg 2006;35:337342.

6. Weber C, Dommerich S, Pau HW, Kramp B. Limited mouth opening after primary therapy of head and neck cancer. Oral Maxillofac Surg 2010;14:169-173.

7. Scott B, Butterworth C, Lowe D, Rogers SN. Factors associated with restricted mouth opening and its relationship to healthrelated quality of life in patients attending a Maxillofacial Oncology clinic. Oral Oncol 2008;44:430-438.

8. Wang CJ, Huang EY, Hsu HC, Chen HC, Fang FM, Hsiung CY. The degree and time-course assessment of radiation-induced trismus occurring after radiotherapy for nasopharyngeal cancer. Laryngoscope 2005;115:1458-1460.

9. Plotz PH, Rider LG, Targoff IN, Raben N, O'Hanlon TP, Miller FW. NIH conference. Myositis: immunologic contributions to understanding cause, pathogenesis, and therapy. Ann Intern Med 1995;122:715-724.

10. Goddard G, Mauro G. Temporomandibular disorders. In: Lalwani AK, ed. Current diagnosis \& treatment otolaryngology- head and neck surgery. 3rd ed. New York: McGraw Hill Medical; 2012. pp. 412-413.

11. Garcia J. MRI in inflammatory myopathies. Skeletal Radiol 2000;29:425-438.

12. Adams EM, Chow CK, Premkumar A, Plotz PH. The idiopathic inflammatory myopathies: spectrum of MR imaging findings. Radiographics 1995;15:563-574.

13. Bensadoun RJ, Riesenbeck D, Lockhart PB, Elting LS, Spijkervet FK, Brennan MT. A systematic review of trismus induced by cancer therapies in head and neck cancer patients. Support Care Cancer 2010;18:1033-1038. 